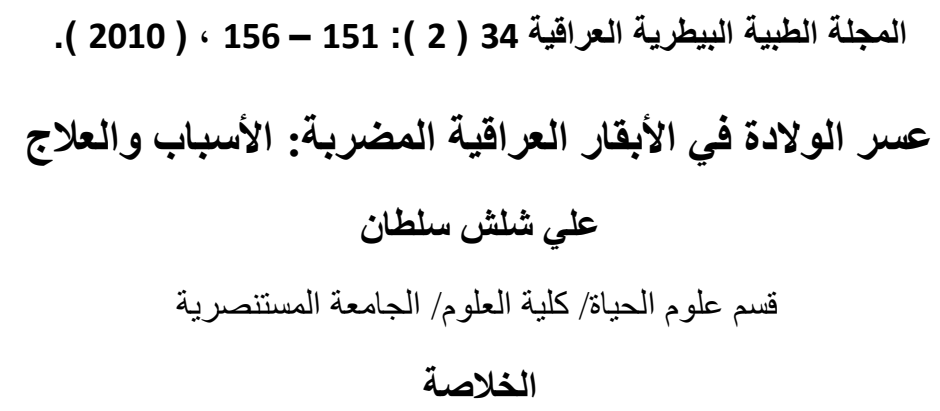

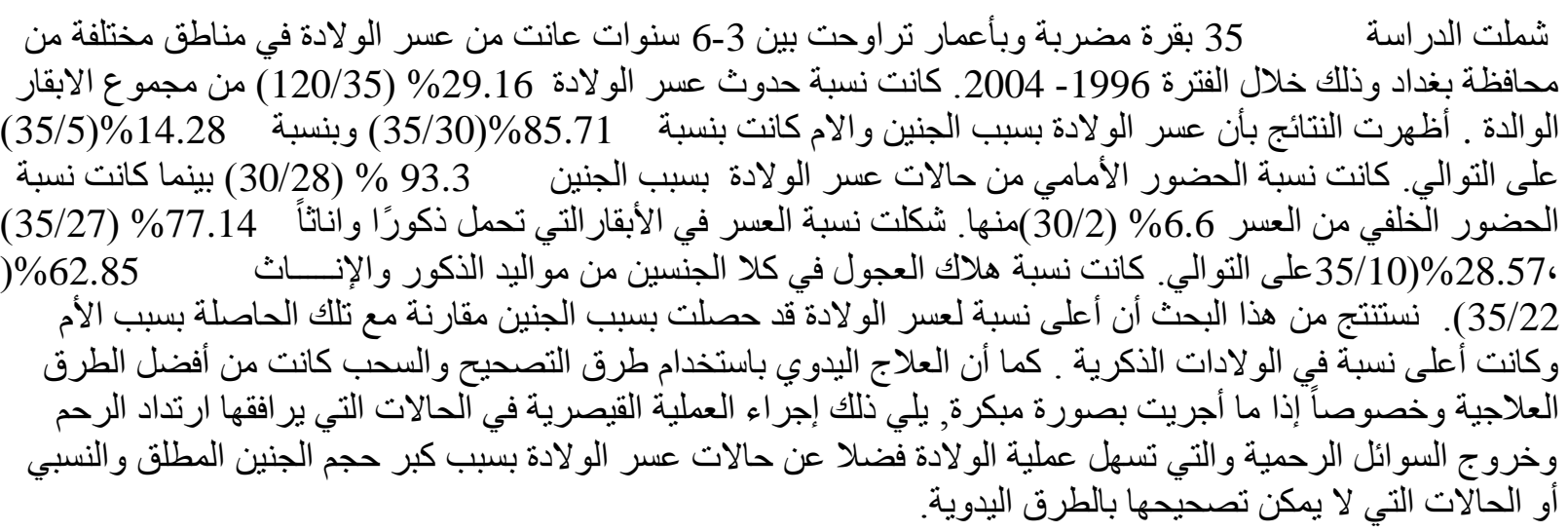

\title{
Dystocia In Iraqi Cross Breed cows: causes and Treatment
}

\author{
Ali Shalash Sultan \\ Department of Biology, College of science ,Almustansyria university
}

Accepted- $\quad / 2010$.

Summary

The study was conducted on 35 Cross breed cows. Three-Six years old suffering from dystocia in various regions of Baghdad province during 1996-2004. The incidence of dystocia was $29.16 \%$ (35/120) from total calving cows. The result showed that the dystocia due to fetal and the dam were $85.71 \%(30 / 35)$ and $14.28 \%(5 / 35)$ respectively. The anterior presentation percentage from fetal dystocia cases was $93.3 \%(28 / 30)$ while the percentage of posterior presentation was $6.6 \%(2 / 30)$ from these cases. Cows delivered male and female calves were formed $77.14 \%(27 / 35)$ and $28.57 \%(10 / 35)$. The calves mortality percentage in both sex was $62.85 \%(22 / 35)$.

The conclusion suggested that high incidence of dystocia due to fetus was in cows calving male calves .The manual repairing, correction and traction was the best methods when performed early, while Cesarean section was the pervecit especially in the cases accompanied with uterine involution and expulsion of uterine fluids that facilate parturition, as well as fetal over size or that can't be correct manually. 
أنشار عدد من الباحثين إلى أن عسر الولادة ( Dystocia ) يحدث أما بسبب الجنين, الأم أو أسباب اخرى ( 1 1, 2, 3, 4 4)

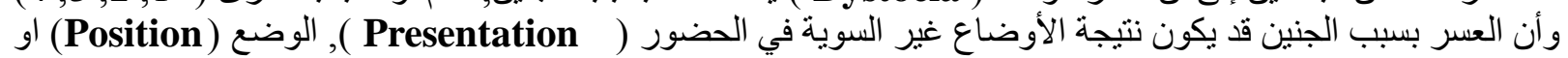

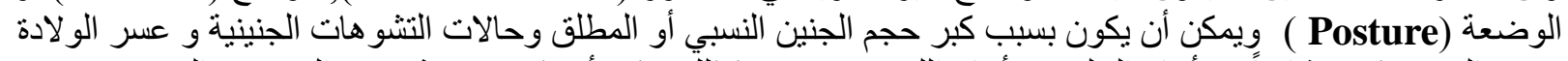

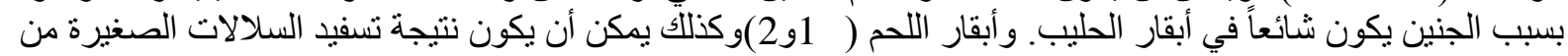

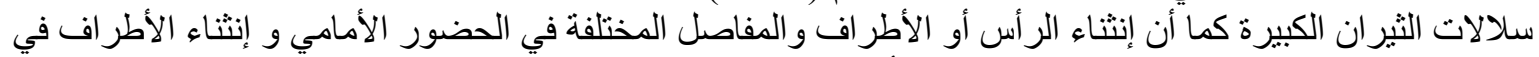

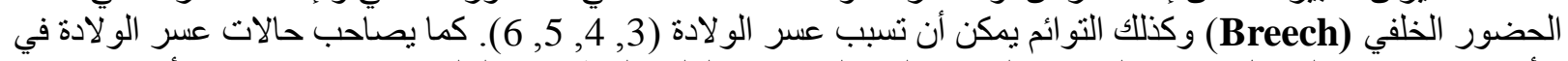

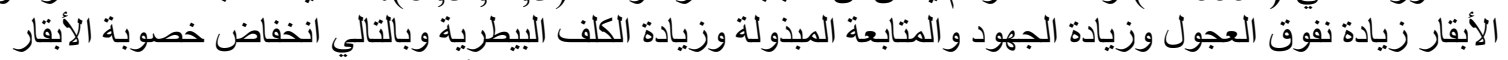

المستقبلية ولربما استبعادها من القطيع (

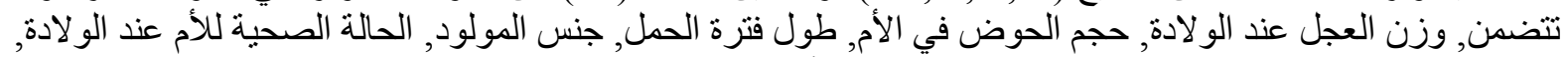

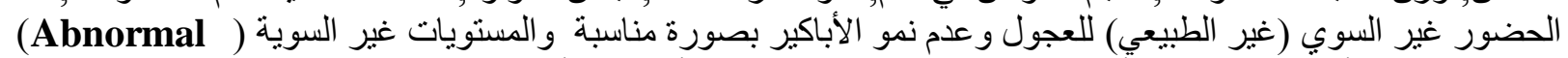

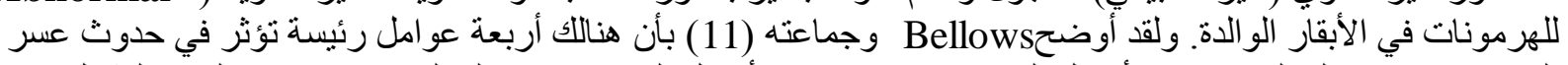

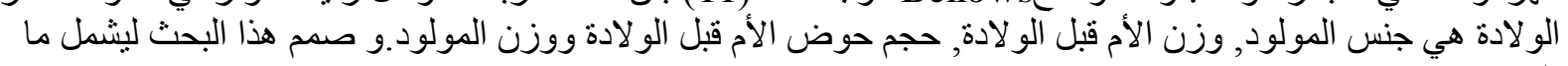
بلي:-

$$
\begin{aligned}
& 1 \text { - - بيان أسباب عسر الو لادة في الأبقار العراقية المضربة والتي تحدث أما بسبب الجنين أو الأم. }
\end{aligned}
$$

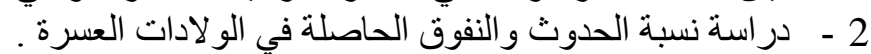

$$
\begin{aligned}
& 3 \text { - استخدام الطرق المختلفة والمتبعة حديثا في معالجة عسر الولادة لادة لاسيما الهرمونية. }
\end{aligned}
$$

\section{المواد وطر ائق العمل}

أجريت الدر اسة على 35 بقرة عر اقية مضربة من مجموع 120 بقرة والدة في مناطق مختلفة من محافظة بغداد وبأعمار

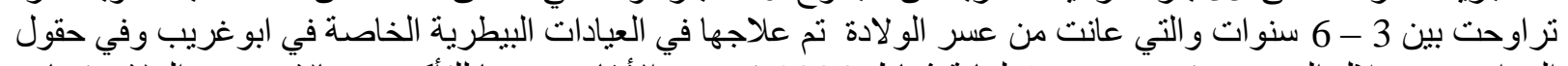

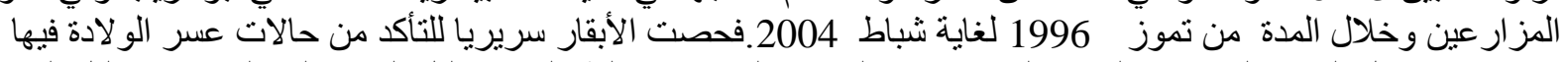

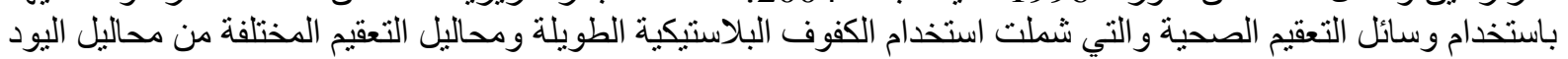

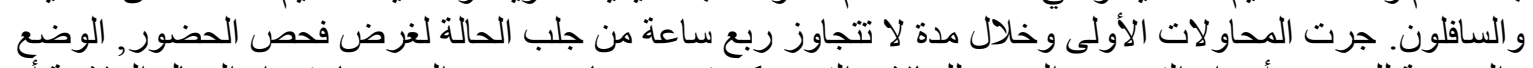

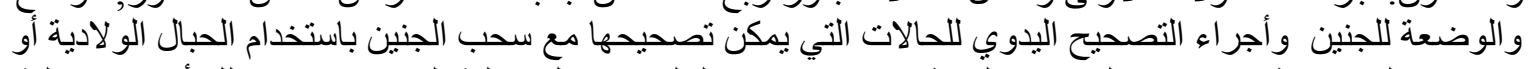

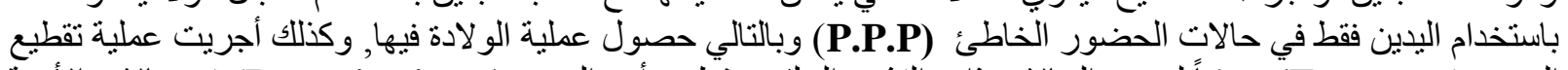

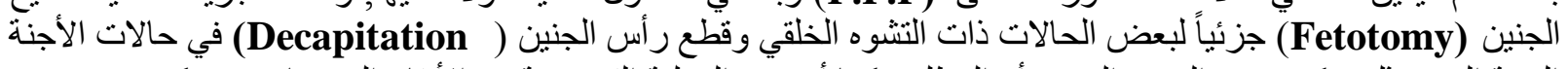

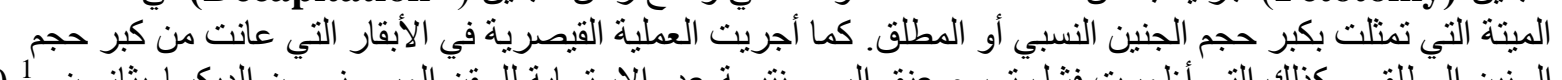

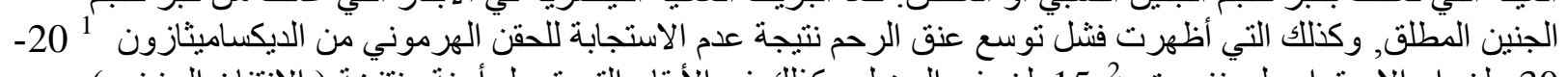

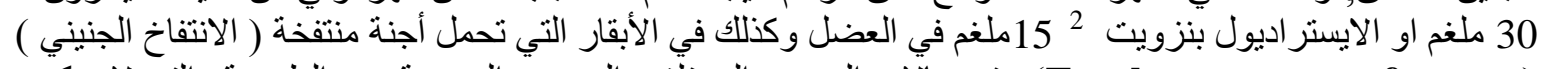

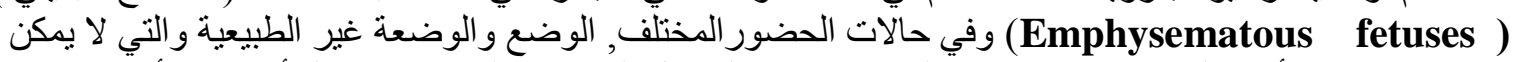

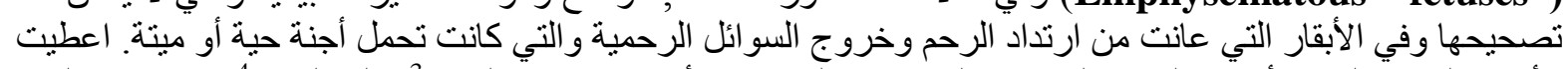

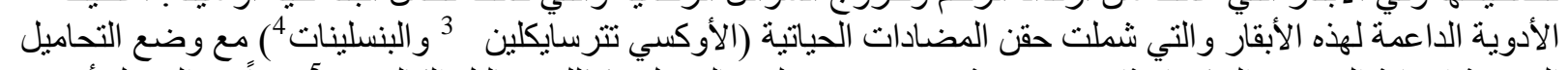

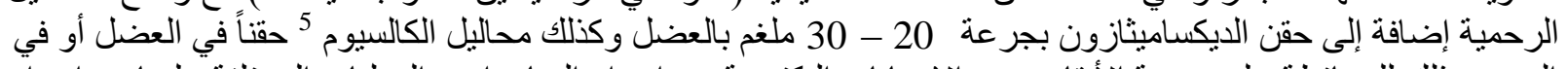

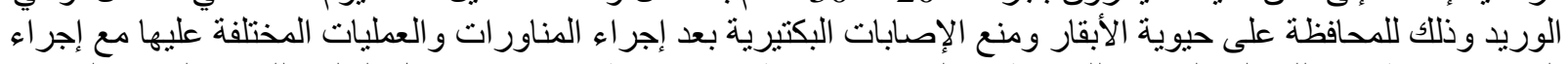

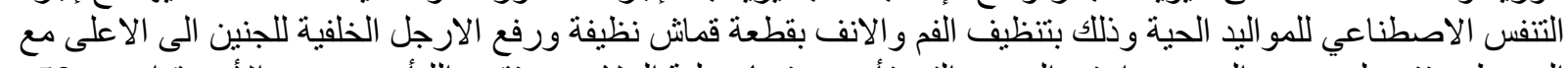

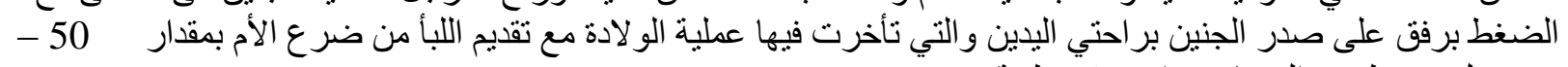
100 مل عن طريق الفم باستخدام محقنه طبية.

1. Dexamethasone $0.2 \%$ ( $2 \mathrm{mg} \backslash \mathrm{ml}$ ) ACDIVET, Acdiema, Damascus.

2. Estradiol benzoate ( $2 \mathrm{mg} \backslash \mathrm{ml}$ ) Intervet International B. V. Boxmeer Holland.

3. Tetroxy 20\% Bimeda chemicals Export, Dublin, Irland.

4. Pencillin G suspension (200.000 Iulml ) Invesa, Industrial veterinaria, S.A. Barcelona, Espanola.

5. Calci ject $40 \%$ Norbrook for hypocalcemia in cattle. 
النتائج

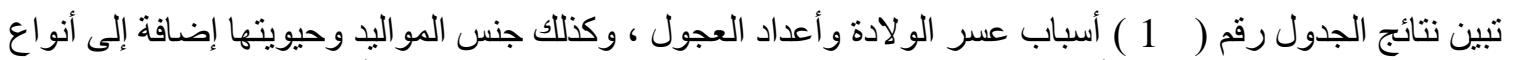

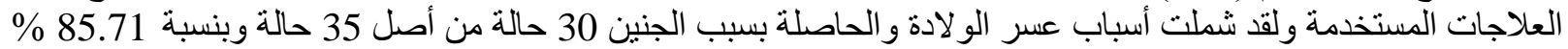

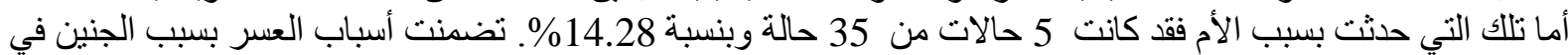

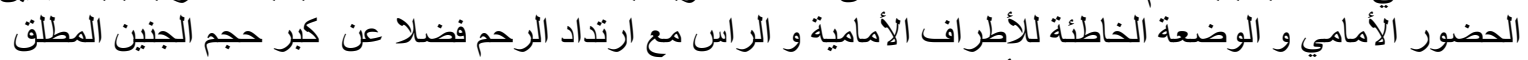

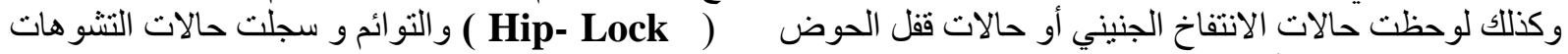
الخلقية مع كبر حجم رأس الجنين(Hydrocephalus) وقد شكلت هذه الحان الحالات نسبة عسر و لادة بلغت

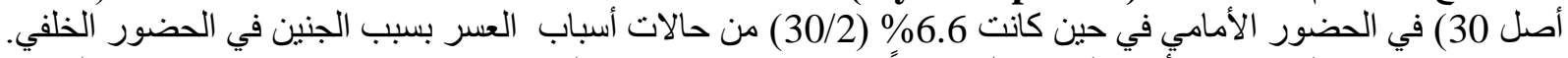

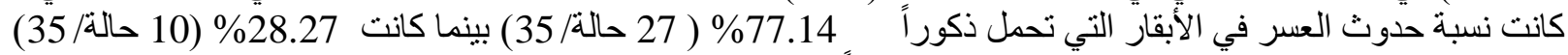

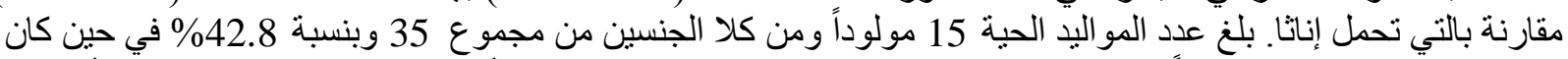

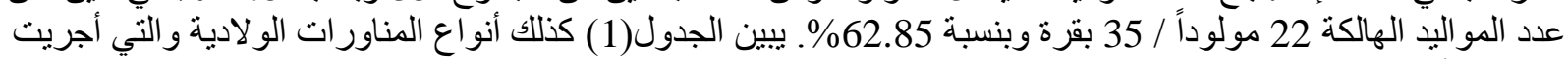

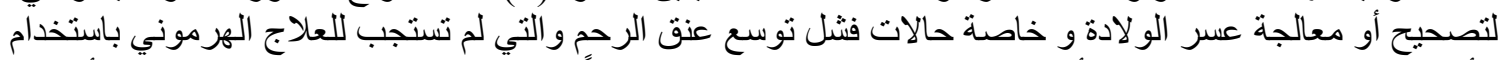

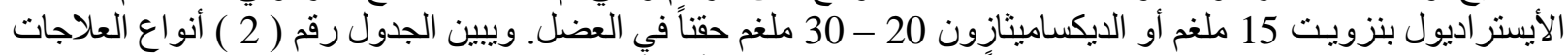

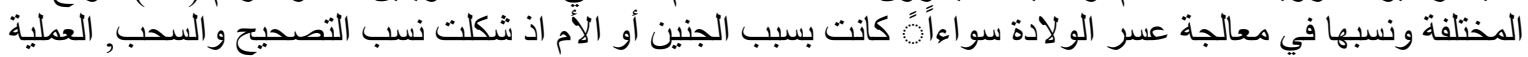

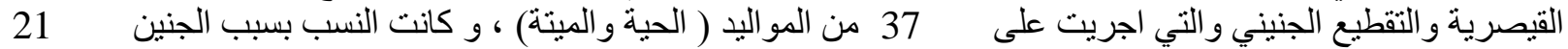

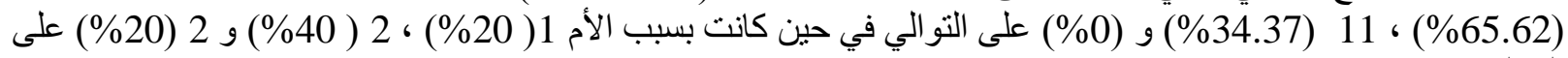
التوالي.

جدول رقم (1) يوضح أسباب عسر الولادة في الابقار المضربة العراقية (المتعقة بالجنين والأم) وأعدادها وحيوية المواليد وجنسها مع العلاجات المختلفة

\begin{tabular}{|c|c|c|c|c|c|c|c|}
\hline \multirow[t]{2}{*}{ العلاجات المستخذمة لعسر الولادة } & \multicolumn{2}{|c|}{ حيوية المواليد } & \multicolumn{2}{|c|}{ جنس المواليا } & \multirow{2}{*}{ علد حالات } & \multirow[t]{2}{*}{ أسباب عسر الولادة } & \multirow[t]{2}{*}{$ت$} \\
\hline & 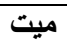 & حي & إناث & ذكور & & & \\
\hline التصحيح مع السحب(4حالات) & 2 & 2 & 0 & 4 & 4 & 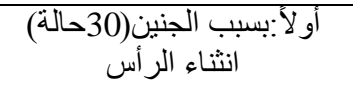 & -1 \\
\hline السحب(8 حالات) و و4حالات) عملية & 7 & 5 & 5 & 7 & 12 & 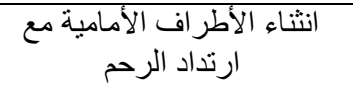 & -2 \\
\hline (حالتان) و عملية قيصرئية (3 حالات) & 4 & 1 & 0 & 5 & 5 & كبر حجم الجنين المطلق & -3 \\
\hline العملية القيصرية & 2 & 0 & 0 & 2 & 2 & الانتفاخ الجنيني & -4 \\
\hline تصحيح وسحب & 0 & 1 & 0 & 1 & 1 & Hip Joint Lock ققل الحوض & -5 \\
\hline تصحيح و سحب (4 حالات) & 4 & 0 & 1 & 3 & 2 & التو ائم Twinning & -6 \\
\hline تصحيح و سحب حالتان & 0 & 2 & 1 & 1 & 2 & حضور خلفي(Breech) & -7 \\
\hline عملية قيصرية حالتان & 2 & 0 & 0 & 2 & 2 & $\begin{array}{l}\text { حالات تشو هات خلقية } \\
\text { (Hydrocephalus) }\end{array}$ & -8 \\
\hline تقطيع الجنين جزئياً و التصحيح و & 1 & 1 & 1 & 1 & 2 & 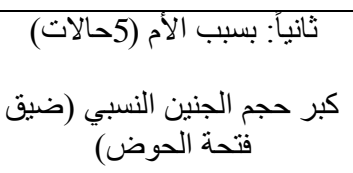 & -1 \\
\hline تصحيح وسحب & 0 & 1 & 0 & 1 & 1 & التو اء الرحم & -2 \\
\hline علاج هرموني و عملية قيصرية & 0 & 2 & 2 & 0 & 2 & فنثل توسع عنق الرحم & -3 \\
\hline & 22 & $15 * *$ & 10 & $27 * *$ & $35^{*}$ & المجموع & \\
\hline
\end{tabular}

*: عدد حالات عسر الو لادة الكلي (بسبب الجنين+الأم) هي35 حالة ذ**: أعداد المو اليد الكلي37 مولود وذلك لوجود حالتين تو أميه من35 حالة 
جدول رقم (2) يوضح أنواع العلاجات المختلفة التي أجريت على عسر الو لادة في الابقار المضربة العر اقية ونسبها المئوية والحاصلة بسبب الجنين و الأم.

\begin{tabular}{|c|c|c|}
\hline بسبب الأم & بسبب الجنين & \\
\hline أعداد المواليد 5 & أعداد المواليد 32 & \\
\hline 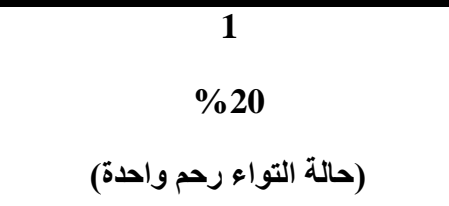 & $\begin{array}{c}21 \\
\% 65.62\end{array}$ & التصحيح مع السحب \\
\hline 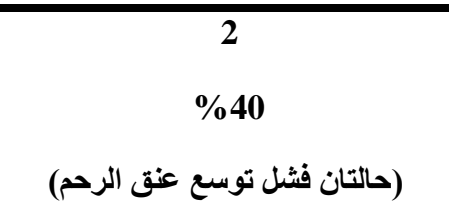 & $\% 34.37$ & جزئي للجنين القيصرية ومن ضمنها حالتّان تقطيع \\
\hline $\begin{array}{c}2 \\
\text { \% 40 } \\
\text { (حالتان بسبب كبر حجم الجنين النسبي) }\end{array}$ & $\begin{array}{c}\mathbf{0} \\
\% \mathbf{0}\end{array}$ & التقطيع الجزئي للجنين لوحده فقط \\
\hline 5 & 32 & المجموع \\
\hline
\end{tabular}

\section{المناقثة}

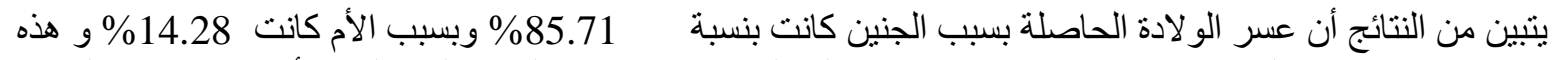

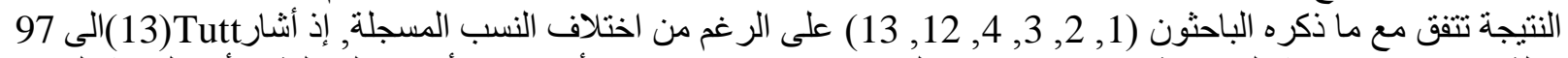

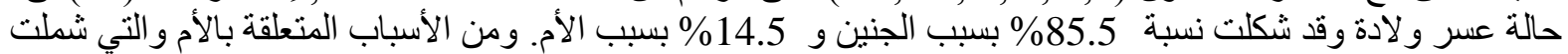

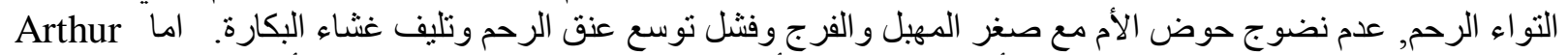

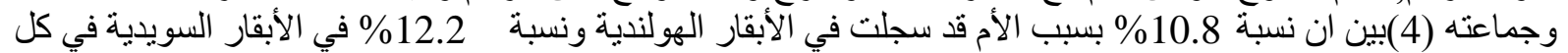

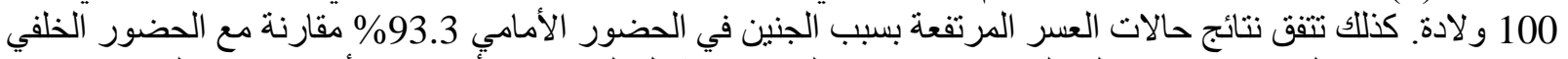

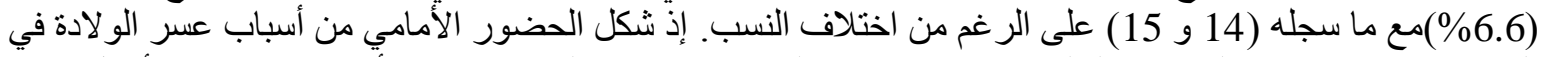

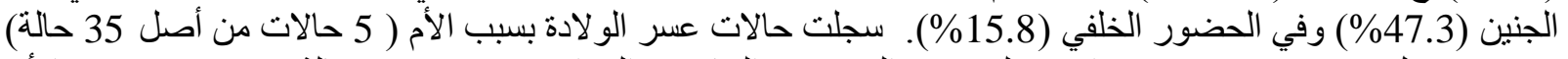

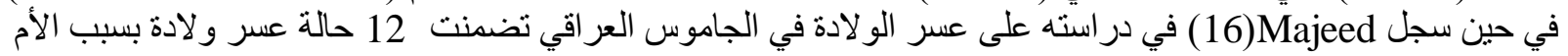

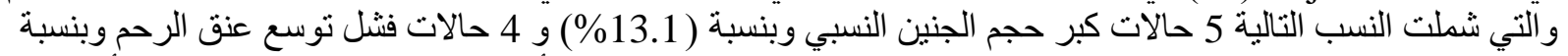

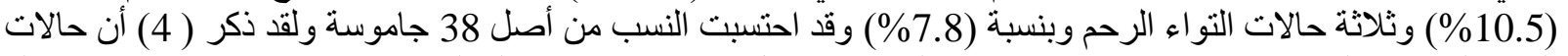

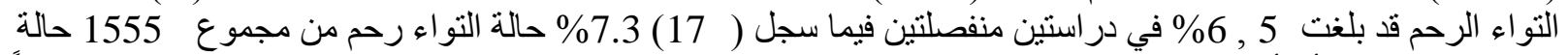

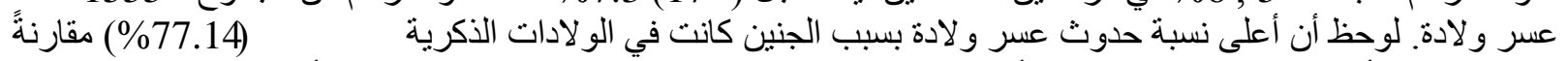

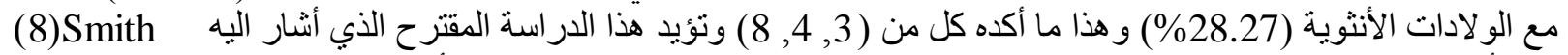

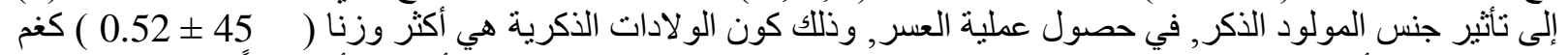

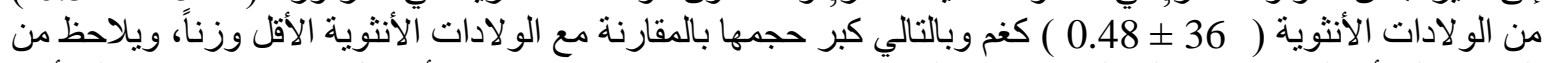
الدراسة إلى أن الهاكاكات في المو اليد وفي كلا الجنسين كانت بنسبة (

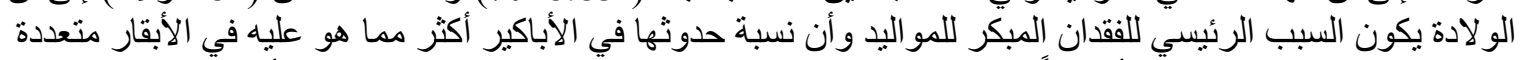

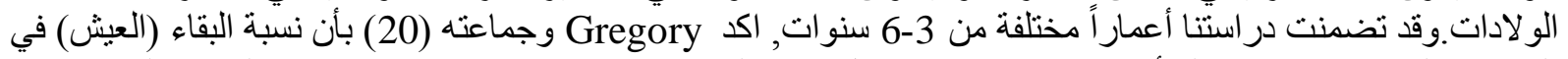

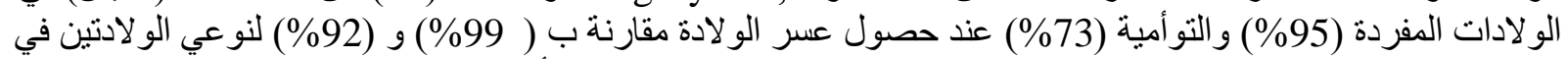

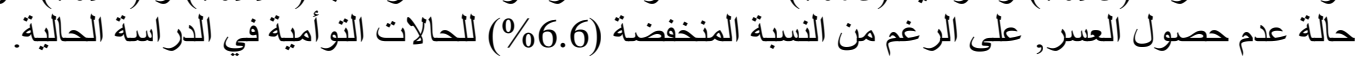

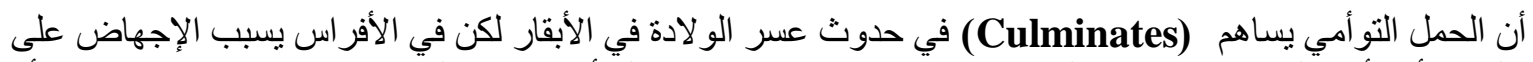

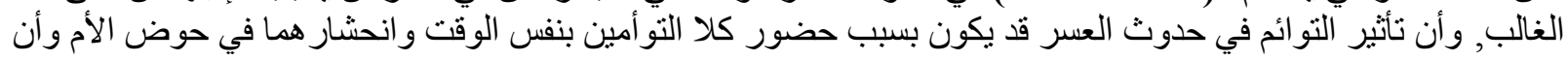


أحد التو ائُ يتقدم داخل القناة الو لادية لكنه لا يولد بسبب أخطاء في (P.P.P أو بسبب و هن الرحم و الحالة التي حصل فيها

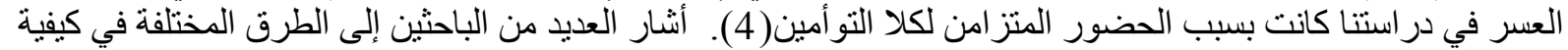

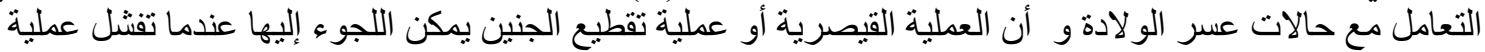

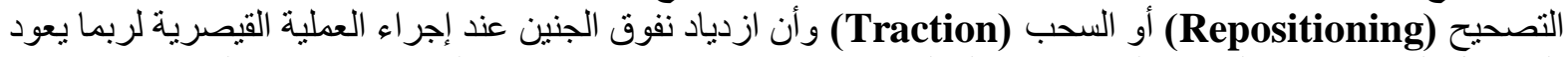

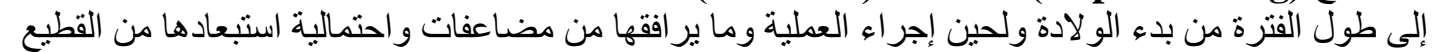

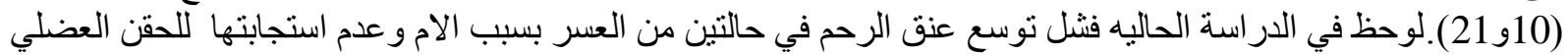

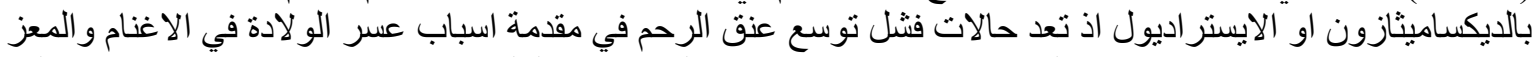

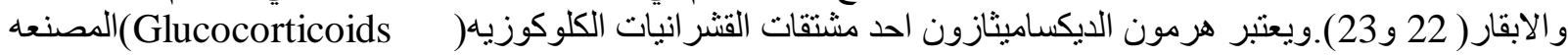

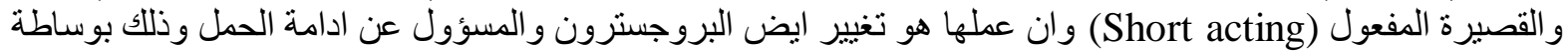

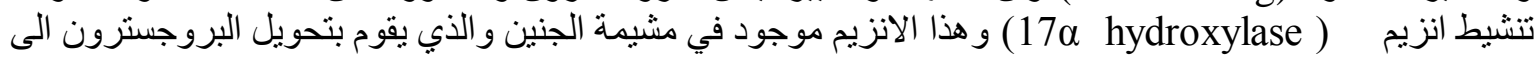

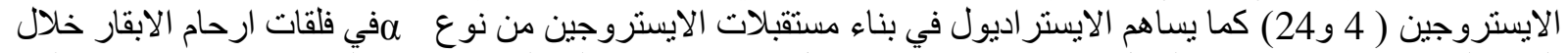

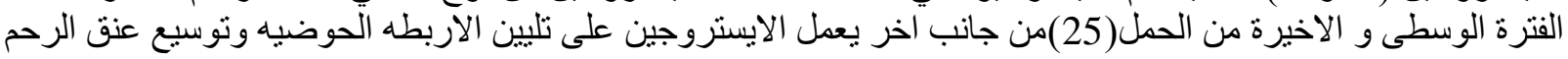

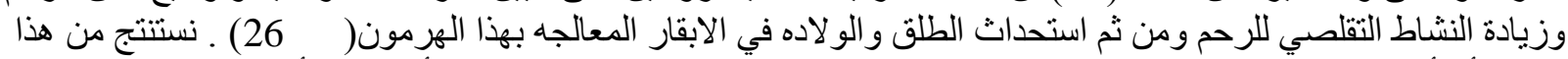

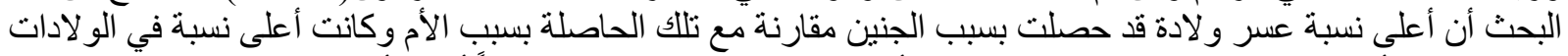

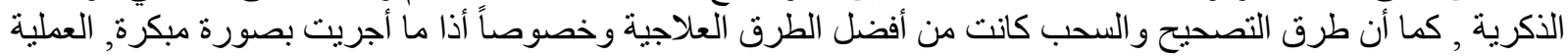

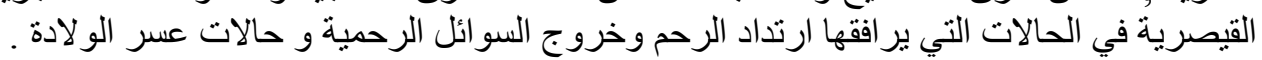

\section{المصادر}

1- Price T Dand JN Witt bank(1978).. Dystocia in Cattle: A Review and implications. Theriogenology 9: 195- 219

2- Jainudeen MR and Hafez. ESE(1980). Reproductive Failure in Females. In Reproduction in farm animals, $4^{\text {th }}$ ed, Edited by Hafez, ESE Lear \& Febiger Philadelphi PP449-470 Roberts SJ(1986). Veterinary Obstetrics and Genital Diseases. $3^{\text {rd }}$ ed. Published by author Woodstock Vermont. PP 227-237.

3- Arthur GH Noakes DE and Pearson H(1996). Veterinary Reproduction and obstetrics $7^{\text {th }}$ ed. London Bailliere Tindall PP 161-182

4- Salma MD King ME Odde KG and Mortimer RG(1991). Costs of veterinary services and vaccines drugs used for prevention and treatment in 60 Tennessee cow-calf operations (19871988) J Am Vet Med Ass 198(8) 1334-1340

5- Johanson JM Berger PJ Kirkpatrick BW and Dentine MR(2001). Twinning Rates for North American Holstein sires J Dairy Sci 84 (9) 2081-2088

6- Detutscher GH(1991). Pelvic Measurements for Reducing Calving Difficulty Neb Guide G87839-A Cooperative Extension, University of Nebraska Lincoln.

7- Smith JW(2005).Correlation of pelvic shape and birth Weight EPDs in reducing Dystocia in Beef cattle Master of science. Agricultural Operations Management December

8- Erb HN Martine SW Ison N and Swaminathau S (1981). Interrelations ships between production and reproductive diseases in Holstein Cows Conditional relationships between production and disease J Dairy sci. 64: 272-281

10- Barkema HW Schukken YH Guard CL Brand A and Vander weyden, GC (1992). Fertility production and culling following Cesarean section in Dairy Cattle. Theriogenology 38: 589599.

11- Bellows RA Genho pc Moore SA and Chase CC (1996). Factors affecting Dystocia in Brahman - Cross heifers in subtropical Southeastern United States J Anim Sci 74:1451-1456.

12- Berger PJ Cubas AC Koehler KJ and Healey MH (1992). Factors affecting Dystocia and early calf mortality in Angus Cows and Heifers. J Anim Sci 70 (6) 1775-1786.

13- Tutt JB (1944) Dystocia in cows Vet J 100154 Cited by Arthur GH et.al. (1996).

14- Sloss V and Duffy J H (1980). Handbook of bovine obstetrics Baltimore: Williams and Wilkins 98 PP 15-16 


\section{المجلة الطبية البيطرية العراقية 34 ( 2 ): 151 - 156 ، ( 2010 ).}

15- Majeed AF Taha MB and Azawi OI (1992). Cesarean section in a local breed Iraqi cattle Iraqi J Vet Sci5:170-18

16- Majeed AFA (2001).Clinical study on Dystocia in Iraqi Buffaloes. Iraqi J Vet Sci 14(1): 125-128.

17- Roberts SJ Veterinary obstetrics and Genital Diseases (1971). $2^{\text {nd }}$ ed. Published by author Woodstock Vermont.PP 330-335

18- Basarab JA Rutter L M and Day PA (1993). The Efficacy of predicting Dystocia in yearling Beef Heifers 11 using Discriminant Analysis J Anim. Sci 71 : 1372-1380

19- Boyles S(2000). Integrated Heifer Management critical success Factors. Ohio State University Extension

20- Gregory KE Echtern Kamp SE Dickerson GE and VanVelek LD (1990). Twinning in cattle III Effects of twinning on dystocia,reproductive traits, Calf survival, calf growth and Cow productivity J Anim Sci. 68 (10): 3133-3144

21- Hobben D Mijten P and de Kruit A (1997) .Factors Influencing complications During Cesarean section on the standing cow. Vet Quarterly 19 88-92

22-Majeed AFand Taha MB(1988). Clinical treatment of Ring womb in Awassi ewes Mesoptamia JAgric.20:53-58.

23-MacDiarmid SC(1983).Induction of parturition in cattle using Corticoids.Areview.Part1Anim Breed Abstracts 51:403-419.

24-Parkinson T.(1993).Induction of parturition in cattle.In practice $\mathrm{J}$ of postgraduate V.15No.3 PP135-137

25-SchulerGw Wirth CH Teichmanu Uand Hoffman B (2002). OccurrenceOf estrogen receptors $\alpha$ in Bovine Placentomes Throught mid and lat Gestation and at parturition.Biology of Repr.66:796798

26-Wood CE(1999). Control of parturition in Ruminant J Repro.Fert. 54:115-126. 